\title{
Antibacterial Susceptibility Testing of Cutibacterium acnes in Acne Vulgaris Patients
}

\author{
Ingus Skadins' \\ Aleksejs Zavorins $\mathbb{1 D}^{2}$ \\ Juta Kroica (D) \\ Terēze Pavloviča $\mathbb{D D}^{3}$ \\ Dagnija Bruzgule ${ }^{3}$ \\ Tatjana Averjanova $\mathbb{D}^{3}$ \\ 'Riga Stradinš̌ University, Department of \\ Biology and Microbiology, Riga, LV-I007, \\ Latvia; ${ }^{2}$ Rīga Stradiňš University, \\ Department of Dermatoveneorology, \\ Riga, LV-I0I0, Latvia; ${ }^{3}$ Riga Stradinš \\ University, Faculty of Medicine, Riga, LV- \\ 1007, Latvia
}

\begin{abstract}
Introduction: Combination therapy is widely used for the treatment of acne vulgaris (AV), including local anti-inflammatory drugs containing antimicrobials, such as clindamycin or erythromycin, to inhibit Cutibacterium acnes (C. acnes) growth and at the same time reduce the production of inflammatory mediators. The aim of the study is to compare the antibacterial susceptibility of $C$. acnes to clindamycin and erythromycin in AV patients compared with healthy patients in the control group (CG).
\end{abstract}

Methods: The prospective study included 56 patients with clinically diagnosed AV symptoms and 12 patients were included in the CG who did not have AV. In the AV group, patient specimen was contents of pustules obtained by squeezing pustules, but in the CG, the specimen was content of sebaceous glands. All specimens were cultivated on a combined Mueller-Hinton solid medium. Identification was done by VITEK2 and followed by determination of antibacterial susceptibility of the isolated $C$. acnes strains by E-test.

Results: $C$. acnes was isolated from samples of 28 (50\%) in the AV group, whereas in the $\mathrm{CG}$, C. acnes was isolated from 10 samples $(80 \%)$. Resistance to clindamycin in both groups was similar, in $6(21.4 \%)$ samples from patients in the AV group and in $2(20.0 \%)$ samples in the $\mathrm{CG}$, but resistance to erythromycin in the $\mathrm{AV}$ patients was higher compared to the $\mathrm{CG}$, in $8(28.6 \%)$ and $1(10 \%)$ accordingly.

Conclusion: Patients with AV have higher rates of resistance to erythromycin than the $\mathrm{CG}$, while resistance to clindamycin is comparable. Resistance data showed no statistically significant association between use of erythromycin and clindamycin and the development of resistance. More $C$. acnes were identified in the CG than in the AV group.

Keywords: acne vulgaris, Cutibacterium acnes, clindamycin, erythromycin, antibacterial susceptibility

\section{Introduction}

Acne vulgaris is a common, chronic, inflammatory skin disease, primarily localised on the face, neck and back. Clinically, AV manifests with the following lesions: comedones, papules, pustules, nodules, cysts. Scarring is also usually present due to dermal inflammation in the aforementioned areas. In approximately $85 \%$ of the cases, acne vulgaris develops in patients aged 12-24 years. ${ }^{1}$ Persistent AV usually starts in puberty; however, the disease continues into adulthood ( $>25$ years of age), persists in $41 \%$ of the adult women and is localised in the $U$ zone of the face. ${ }^{2,3}$ Research shows that $12-22 \%$ of the adult women suffer from $\mathrm{AV}^{4}$

Cutibacterium acnes (C. acnes) is a Gram-positive, anaerobic/microaerophilic rod mainly localized in the pilosebaceous unit and constituting $20-70 \%$ of the stratum corneum of the normal microbiota of the skin. ${ }^{5,6}$ The pathogenicity of
Correspondence: Ingus Skadins

Riga Stradiňš University, Department of Biology and Microbiology, Dzirciema iela

I6, Riga, LV-I007, Latvia

$\mathrm{Tel}+37167061570$

Email Ingus.Skadins@rsu.lv 
C. acnes is associated with different ribotypes, it has been observed that ribotypes 4 and 5 are associated with the development of AV. The pathogenicity of C. acnes is also affected by the release of lipases, chemotactic factors and enzymes that stimulate the breakdown of comedones releasing the contents and activating inflammatory cells, keratinocytes, and synthesis of proinflammatory factors. ${ }^{7}$

The typical mechanism of pathogenesis of AV is associated with local hypersecretion of sebum, hyperkeratinisation of the follicular channel, C. acnes colonization, and development of inflammation by activating monocyte TLR-2 receptors and inducing the synthesis of proinflammatory cytokines IL-12 and IL-8. Elevated perifollicular CD4+ T cell counts and IL1 $\alpha$ levels have been shown in inflammatory areas of acne. Literature on the consumption of milk, as well as products with high glycaemic index has shown that these are contributing factors to the development of $\mathrm{AV}^{8,9}$ It has been scientifically proven that amino acids in milk promote insulin secretion and the synthesis of insulin-like growth factor-1 (IGF-1). ${ }^{10,11}$ IGF-1 induces follicular epithelial growth and hyperkeratinisation, and elevated serum levels of IGF-1 correlate with the severity of $\mathrm{AV} .^{12}$

Combination therapy is widely used in the treatment of $\mathrm{AV}$, including local anti-inflammatory drugs containing known antimicrobials, such as clindamycin or erythromycin, to inhibit $C$. acnes growth and at the same time reducing the production of inflammatory mediators. However, doxycycline is prescribed more frequently. ${ }^{13}$ Decreased susceptibility of $C$. acnes strains to antibiotics has been reported in many countries over the past decade, indicating that antibiotic-resistant $C$. acnes is becoming a global problem in acne patients. ${ }^{4,14}$

\section{Objective}

To compare the antibacterial susceptibility of $C$. acnes to clindamycin and erythromycin in acne vulgaris (AV) patients compared with healthy patients in the control group (CG).

\section{Materials and Methods Ethics}

The research was approved by the Ethics Committee of Riga Stradinš̌ University (04.07.2019) (No. 6-2/6/14). The study was conducted in accordance with the Declaration of Helsinki and the study participants signed an informed consent form.
For participants under the age of 18 to be enrolled in the study, the parent or legal guardian provided informed consent on behalf of the child.

\section{Patient Selection}

The prospective study included 68 patients from November 2017 to January 2020. The study includes AV group and CG. The skin condition of patients included in both study groups was clinically evaluated and diagnosis of AV was made by a dermatovenerologist in accordance with European guidelines by assessing the presence of noninflammatory and inflammatory lesions, determining their number, including papules, pustules and deep inflammatory lesions (cysts and nodules) on the face, neck, and chest. All patients from both study groups answered to of a series of questions related to previous antibiotic usage, way of usage and skin care.

\section{Acne Vulgaris Group}

AV group included 56 patients with clinically diagnosed acne vulgaris symptoms (open or closed comedones, papules, pustules, or cystic lesions).

Inclusion criteria in the acne vulgaris group:

Grade 1 - comedonal acne (closed or open comedones)

Grade 2 - mild to moderate papulopustular acne

Grade 3 - severe papulopustular acne

Grade 4 - severe nodular acne, acne conglobate

Exclusion criteria in the AV group were defined as other skin conditions such as rosacea, perioral dermatitis or other manifestations of infection in acne-affected skin.

\section{Control Group}

The CG included 12 healthy individuals with no previous history of Acne vulgaris or other skin condition. Exclusion criteria in the CG included use of topical or systemic antibacterial therapy in the previous month, acne vulgaris or other chronic inflammatory skin diseases, polycystic ovary syndrome.

\section{Collection and Transportation of Patient Specimen}

In the AV group, the selected patient specimen was pus (contents of pustules) obtained by squeezing pustules. Prior to specimen collection, the area surrounding the skin lesion on the face ( $\mathrm{T}$ zone, forehead, cheeks, chin) was disinfected with an alcohol pad (70\%). 
The specimens were transported in AMIES (Medical Wire \& Equipment, England) transport medium to the laboratory within 2 hours. In the CG, specimens were collected by obtaining contents of sebaceous glands after local disinfection of the skin around the nose. Specimens were transported in AMIES transport medium to the laboratory within 2 hours.

\section{Cultivation and Separation of Material}

$C$. acnes was cultivated on a combined Mueller-Hinton solid medium (E\&O Laboratories; Liofilchem) under anaerobic conditions for 5 days at $37^{\circ} \mathrm{C}$ (Oxoid TM AnaeroGen тм, $7-15 \% \mathrm{CO} 2)$.

\section{Microscopy}

Selection criteria for VITEK included growth of $C$. acnes on blood agar and formation of small white colonies on $\mathrm{MH}$ medium. These colonies were selected for microscopy and stained using the Gram staining technique. C. acnes are $\mathrm{Gr}+$ rods; therefore, VITEK identification was performed following microscopic visualization of $\mathrm{Gr}+$ rods.

\section{Identification and Determination of Antibacterial Susceptibility}

C. acnes was identified by VITEK2 ANC ID cards (bioMérieux) after preparation of bacterial suspensions with different optical densities from 2.7 to 3.3 according to McFarland. Antibacterial susceptibility testing was performed using commercial E-tests for clindamycin and erythromycin with determination of MIC (minimum inhibitory concentration) in the identified $C$. acnes samples. Bacterial suspension for antibacterial susceptibility testing was prepared according to the manufacturer's recommendations with an optical density of 0.5 McFarland. Cultivation was performed using combined blood agar and Mueller-Hinton solid medium under anaerobic conditions for 5 days at $37^{\circ} \mathrm{C}$ (Oxoid ${ }^{\mathrm{TM}}$ AnaeroGen ${ }^{\mathrm{TM}}, 7-15 \%$ $\mathrm{CO} 2$ ). We analysed minimum inhibitory concentrations of erythromycin and clindamycin; a lower MIC means that lower concentrations of antibacterial agents are required to inhibit the growth of $C$. acnes. Strains that did not show any MIC value within the specified range were considered resistant in our study.

\section{Statistical Data Processing}

Data processing was performed using Microsoft Excel 2013 and IBM SPSS Statistics 26.0.
For quantitative data, assumptions were tested, including whether they were subject to normal distribution by using the Shapiro-Wilk test. Appropriate statistical test was then selected. To determine if there is a difference in the age distribution between groups, the Mann-Whitney $U$-test was used. Fisher's exact test was used for qualitative data to determine statistically significant associations. A $p$ value of $<0.05$ was considered statistically significant.

\section{Results}

Pustular samples were obtained from 56 patients with AV and 12 CG. Patients' age ranged from 12 to 35 years, with a median age of 22 years (interquartile range 20-25 years), of which 49 were female and 19 were male.

AV patients were aged 12 to 35 years, median age 22 years (IQR $=20-25$ years), of which 49 were women and 17 men. In the acne group, $22(39.2 \%)$ patients had acne comedonica, 25 (44.6\%) had acne papulopustulosa moderate form, acne papulopustulosa severe form was diagnosed in $8(14.2 \%)$ and acne nodulocystica was found in 1 $(1.7 \%)$ of the patients. Systemic clindamycin was used by 2 (3.5\%); erythromycin by $2(3.5 \%)$; doxycycline by 6 $(10.7 \%)$ and other antibiotics by $6(10.7 \%)$ patients.

The study participants also used topical antibacterial agents, 5 (8.9\%) patients used an agent containing clindamycin and $3(5.3 \%)$ used one that contained erythromycin. Fourteen $(25 \%)$ patients used agents that did not contain any of the previously mentioned active substances and 44 (78.5\%) patients, according to their medical history, did not use any topical agents.

Analysis of skin care habits showed that $45(80.3 \%)$ or most participants used skin cleansing products twice a day, $15(26.7 \%)$ did not use any products, 4 (7.1\%) used 1 product daily and $2(3.5 \%)$ participants used a product more than twice a day.

\section{Analysis of Results by Group}

Of the 56 patients with $\mathrm{AV}, C$. acnes was isolated from samples of $28(50 \%)$ patients and no growth was detected in samples of $28(50 \%)$ patients, whereas in the $\mathrm{CG}$, comprised of 12 individuals, $C$. acnes was isolated from $10(80 \%)$ samples.

In regards to use of oral antibiotics, erythromycin was taken by $1(3.6 \%)$ patient in the group with growth of C. acnes (group 1) and in $1(3.6 \%)$ patient in the AV group without growth of $C$. acnes (group 2), amoxicillin was taken by $4(14.3 \%)$ patients in group 1 and $2(7.1 \%)$ patients in group 2 . No patients $(0.0 \%)$ used clindamycin 
in group 1, yet $2(7.1 \%)$ patients used it in group 2, and doxycycline was used by $4(14.3 \%)$ patients in group 1 and by $2(7.1 \%)$ in group 2 . No participants in the CG used antibiotics (according to the study exclusion criteria).

In the $\mathrm{AV}$ group, median age was 22 years $(\mathrm{IQR}=$ 19.5-25.8). In the $\mathrm{CG}$, median age was 20 years $(\mathrm{IQR}=$ 20-21.3). Results of Mann-Whitney $U$-test show that the difference in distribution of patients' age between groups was not statistically significant $(\mathrm{U}=176.5$; $\mathrm{p}=0.230$ ).

Analysis of use of topical agents (Table 1) in the acne group $(n=28)$ showed that $17(60.7 \%)$ patients did not use local antimicrobials; 2 (97.1\%) used a topical agent containing clindamycin; no patients $(0.0 \%)$ used an erythromycin containing agent $(0.0 \%) ; 9(32.1 \%)$ patients used neither erythromycin nor clindamycin.

In the CG $(n=12)$, none of the participants $(0 \%)$ had used topical agents containing clindamycin or erythromycin for treatment of AV.

Analysis of patients $(n=28)$ in the group with no growth or undetectable growth of $C$. acnes and a clinical diagnosis of AV shows that 17 (60.7\%) patients did not use local agents; 3 (10.7\%) used a clindamycin containing topical agent; 3 $(10.7 \%)$ used erythromycin containing agents, while 5
(17.9\%) used topical agents containing neither erythromycin nor clindamycin.

\section{Clindamycin}

In the AV group, none of the respondents used antibacterial agents containing clindamycin; therefore, we cannot conclude whether the possible resistance in these cases is related to previous treatment with clindamycin. In the group with no use of clindamycin, isolates from 22 (78.6\%) patients were sensitive and resistance to clindamycin was observed in $6(21.4 \%)$ patients.

\section{Erythromycin}

With Fisher's exact test, we concluded that there was no statistically significant association between antibiotic use and the development of erythromycin resistance in the AV group ( $\mathrm{p}>0.999)$ (Table 2).

Resistance to erythromycin in the acne group was observed in $0(0.0 \%)$ participants in the erythromycin arm, whereas it was observed in the $8(29.6 \%)$ participants who had not used erythromycin. In the $\mathrm{CG}$, resistance was observed in $1(100 \%)$ participant who did not use erythromycin-containing antibacterial agents (Figure 1.)

Table I Use of Topical Antibacterial Agents

\begin{tabular}{|l|l|l|l|l|}
\hline \multirow{2}{*}{} & \multicolumn{2}{|l|}{ Acne Group (n=56) } & \multicolumn{2}{l|}{ Control Group (n=12) } \\
\cline { 2 - 5 } & $\begin{array}{l}\text { Positive for C. acnes } \\
(\mathbf{n = 2 8 )}\end{array}$ & $\begin{array}{l}\text { Negative for C. acnes } \\
(\mathbf{n}=\mathbf{2 8})\end{array}$ & $\begin{array}{l}\text { Positive for C. acnes } \\
(\mathbf{n}=10)\end{array}$ & $\begin{array}{l}\text { Negative for C. acnes } \\
(\mathbf{n}=\mathbf{2})\end{array}$ \\
\hline Does not use & $17(60.7)$ & $17(60.7)$ & $10(100.0)$ & $0(0)$ \\
Contains clindamycin & $2(7.1)$ & $3(10.7)$ & $0(0.0)$ & $0(0.0)$ \\
Contains erythromycin & $0(0.0)$ & $3(10.7)$ & $0(0.0)$ & $0(0.0)$ \\
Others antibiotics & $9(32.1)$ & $5(17.9)$ & $0(0.0)$ & $0(0.0)$ \\
\hline
\end{tabular}

Table 2 C. acnes Susceptibility to Antibacterial Agents

\begin{tabular}{|c|c|c|c|c|c|c|}
\hline \multirow[t]{2}{*}{ Antibiotics } & \multicolumn{3}{|c|}{ C. acnes Group $(\mathrm{n}=28)$} & \multicolumn{3}{|c|}{ Control Group $(n=10)$} \\
\hline & $\begin{array}{c}\text { Sensitive, } \\
\mathbf{N}(\%)\end{array}$ & $\begin{array}{c}\text { Resistant, } \\
\text { N (\%) }\end{array}$ & p value & $\begin{array}{c}\text { Sensitive, } \\
\mathbf{N}(\%)\end{array}$ & $\begin{array}{c}\text { Resistant, } \\
\text { N (\%) }\end{array}$ & p value \\
\hline $\begin{array}{l}\text { Clindamycin } \\
\text { Using } \\
\text { Not using }\end{array}$ & $\begin{array}{c}0(0.0) \\
22(78.6)\end{array}$ & $\begin{array}{c}0(0.0) \\
6(21.4)\end{array}$ & - & $\begin{array}{c}0(0.0) \\
8(80.0)\end{array}$ & $\begin{array}{c}(0.0) \\
2(20.00)\end{array}$ & - \\
\hline $\begin{array}{l}\text { Erythromycin } \\
\text { Using } \\
\text { Not using }\end{array}$ & $\begin{array}{l}\text { I (100.0) } \\
19(70.4)\end{array}$ & $\begin{array}{c}0(0.0) \\
8(29.6)\end{array}$ & $>0.999$ & $\begin{array}{c}0(0.0) \\
9(90.0)\end{array}$ & $\begin{array}{c}0(0.0) \\
I(10.0)\end{array}$ & - \\
\hline
\end{tabular}

Notes: p value - Fisher's Exact test “-“, not possible to use Fisher's Exact test due to zero patients in the "using” group (there were no patients who used clindamycin therefore no statistical analysis was applied.) 


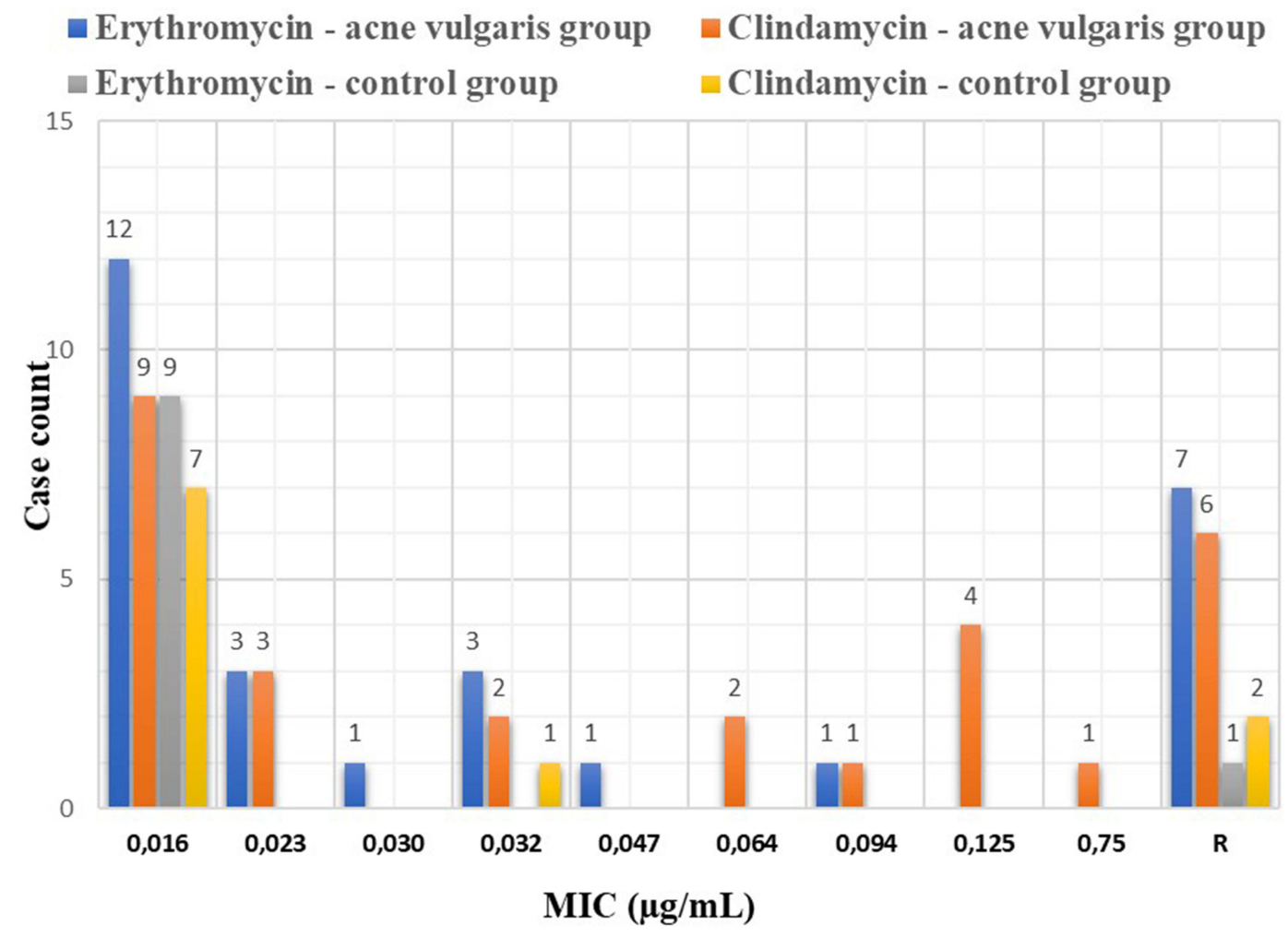

Figure I Minimum inhibitory concentration (MIC) distribution in isolated C. acnes samples. $R$ (resistance) $=$ MIC $>256$ (not detectable).

\section{Discussion}

In our study we found higher resistance to erythromycin in the AV group compared to the CG whereas susceptibility to clindamycin was similar in both groups. A significant association between diagnosis of AV or prior administration of antibiotics and development of resistance was not found $(\mathrm{p}>0.05)$. However, it should be noted that the amount of samples was insufficient, and it is not known whether a statistically significant association would emerge if there were more participants. Upon analysis of the design of our study, we conclude that the groups were not homogeneous. It may be the reason why no association was found between antibiotic use and development of resistance. To improve the study design, it is necessary to create comparable groups in regards to age and gender and to consider the severity of acne.

Furthermore, EUCAST guidelines do not establish clinical breakpoints determining bacterial resistance for $C$. acnes, therefore $C$. acnes were assumed to be resistant when the MIC value was not detectable. Perhaps more precise data regarding susceptibility of $C$. acnes could be acquired if the clinical breakpoints for E-tests were determined or an option to use other methods existed.

In our study, growth rates of $C$. acnes were higher in the $\mathrm{CG}$ compared to the AV group. A possible reason might be that patients in the acne group had received prior treatment and more frequently followed a meticulous skincare regimen potentially eradicating C. acnes, even though we did not find any significant association in our study.

We observed that isolates resistant to clindamycin were also mostly resistant to erythromycin. Other European studies have shown similar results, resistance to erythromycin and clindamycin exceeded $70 \%$ in acne vulgaris patients in France and was as high as $91 \%$ in Spain. ${ }^{4}$ Cross-resistance to both antibiotics is more common and its development correlates with antibiotic-prescribing habits. ${ }^{15}$

When assessing resistance and prior use of antibiotics in the AV group, results of questionnaires showed that none of the respondents had used clindamycin before and only one had used erythromycin; thus, a significant association could not be established. In order to draw conclusions, inclusion criteria should also incorporate previous administration of the aforementioned antibiotics. It would 
also be useful to analyse resistance towards other antibiotics used in the treatment of AV such as doxycycline and minocycline.

In research done by Ross et al, data showed that resistance was observed in individuals who had not previously used antibiotics and in dermatologists specialised in the treatment of acne. Meanwhile, doctors working in other types of outpatient institutions did not have the resistant strains. Interpersonal contact and use of local agents were mentioned as possible reasons. ${ }^{16}$

The development of resistance could also be due to the presence of Staphylococcus aureus (S. aureus) and Staphylococcus epidermidis (S. epidermidis) in the skin microbiota, which a decrease in susceptibility to erythromycin and clindamycin has been observed. ${ }^{17}$

Sari et al reported that the most common bacteria in AV patients are S. epidermidis (47.5\%) and C. acnes $(21.2 \%){ }^{18}$

Study conducted by Jusuf et al concerning microorganisms in non-inflammatory lesions (closed comedones) found eight other bacteria such as $S$. epidermidis (52.5\%), Staphylococcus hominis (12.5\%), Staphylococcus haemolyticus (7.5\%), S. aureus (5.0\%) and others besides C. acnes (17.5\%), whereas increased growth of C. acnes (25.0\%), S. epidermidis (42.5\%), S. hominis (22.5\%), S. aureus (12.5\%) and other bacteria was found in inflammatory lesions. ${ }^{3,19}$ Resistance may be transferred vertically (via plasmids) to S. epidermidis, which in turn may transfer resistance in the same way to S. aureus permitting rapid development of resistance. In C. acnes, resistance develops via vertically transmitted mutational changes, a process that takes longer.

Development of resistance could also be associated with age and disease severity, which can vary among biotypes and phylotypes of C. acnes, and duration of the disease. Another potential mechanism of resistance to erythromycin involves spot mutations in the peptidyl transferase 23S RNA region. ${ }^{15}$

\section{Conclusions}

Patients with AV have higher rates of resistance to erythromycin than the CG, while resistance to clindamycin is comparable. Resistance data showed no statistically significant association between use of erythromycin and clindamycin and the development of resistance. More C. acnes were identified in the $\mathrm{CG}$ than in the $\mathrm{AV}$ group.

\section{Disclosure}

The authors report no conflicts of interest in this work.

\section{References}

1. Skroza N, Tolino E, Mambrin A, et al. Adult acne versus adolescent acne: a retrospective study of 1,167 patients. J Clin Aesthet Dermatol. 2018;11(1):21-25.

2. Zeichner JA, Baldwin HE, Cook-Bolden FE, et al. Emerging issues in adult female acne. J Clin Aesthet Dermatol. 2017;10(1):37-46.

3. Jusuf NK, Putra IB, Sari L. Differences of microbiomes found in non-inflammatory and inflammatory lesions of acne vulgaris. Clin Cosmet Investig Dermatol. 2020;13:773-780. doi:10.2147/CCID

4. Zhang N, Yuan R, Xin KZ, Lu Z, Ma Y. Antimicrobial susceptibility, biotypes and phylotypes of clinical cutibacterium (formerly propionibacterium) acnes strains isolated from acne patients: an observational study. Dermatol Ther. 2019;9(4):735-746. doi:10.1007/ s13555-019-00320-7

5. Grice EA, Kong HH, Conlan S, et al. Topographical and temporal diversity of the human skin microbiome. Science. 2009;324 (5931):1190-1192. doi:10.1126/science. 1171700

6. Beylot C, Auffret N, Poli F, et al. Propionibacterium acnes: an update on its role in the pathogenesis of acne. J Eur Acad Dermatol Venereol. 2014;28(3):271-278. doi:10.1111/jdv.12224

7. Fitz-Gibbon S, Tomida S, Chiu BH, et al. Propionibacterium acnes strain populations in the human skin microbiome associated with acne. J Invest Dermatol. 2013;133(9):2152-2160. doi:10.1038/jid.2013.21

8. Kim J, Ochoa MT, Krutzik SR, et al. Activation of toll-like receptor 2 in acne triggers inflammatory cytokine responses. J Immunol. 2002;169(3):1535-1541. doi:10.4049/jimmunol.169.3.1535

9. Lee SE, Kim JM, Jeong SK, et al. Protease-activated receptor-2 mediates the expression of inflammatory cytokines, antimicrobial peptides, and matrix metalloproteinases in keratinocytes in response to Propionibacterium acnes. Arch Dermatol Res. 2010;302 (10):745-756. doi:10.1007/s00403-010-1074-z

10. Melnik BC. Evidence for acne-promoting effects of milk and other insulinotropic dairy products. Milk Milk Products Human Nutrition. 2011;67:131-145.

11. Mirdamadi Y, Bommhardt U, Goihl A, et al. Insulin and Insulin-like growth factor-1 can activate the phosphoinositide-3-kinase/Akt/ FoxO1 pathway in T cells in vitro. Dermato-Endocrinology. 2017;9 (1):e1356518. doi:10.1080/19381980.2017.1356518

12. Juhl CR, Bergholdt HK, Miller IM, et al. Dairy intake and acne vulgaris: a systematic review and meta-analysis of 78,529 children, adolescents, and young adults. Nutrients. 2018;10(8):1049. doi:10.3390/nu10081049

13. Nast A, Dréno B, Bettoli V, et al. European evidence-based (S3) guideline for the treatment of acne-update 2016-short version. J Eur Acad Dermatol Venereol. 2016;30(8):1261-1268. doi:10.1111/jdv.13776

14. Zhu T, Zhu W, Wang Q, et al. Antibiotic susceptibility of Propionibacterium acnes isolated from patients with acne in a public hospital in Southwest China: prospective cross-sectional study. $B M J$ Open. 2019;9:e22938. doi:10.1136/bmjopen-2018-022938

15. Schafer F, Fich F, Lam M, et al. Antimicrobial susceptibility and genetic characteristics of Propionibacterium acnes isolated from patients with acne. Int $J$ Dermatol. 2013;52(4):418-425. doi:10.1111/j.1365-4632.2011.05371

16. Ross JI, Snelling AM, Carnegie E, et al. Antibiotic-resistant acne: lessons from Europe. $B r \quad J$ Dermatol. 2003;148(3):467-478. doi:10.1046/j.1365-2133.2003.05067.x

17. Alkhawaja E, Hammadi S, Abdelmalek M, et al. Antibiotic resistant Cutibacterium acnes among acne patients in Jordan: a cross sectional study. BMC Dermatol. 2020;20(1):17. doi:10.1186/s12895-02000108-9

18. Sari L, Jusuf N, Putra I. Bacterial identification of acne vulgaris. Bali Med J. 2020;9(3):753-756. doi:10.15562/bmj.v9i3.1737

19. Jappe U. Pathological mechanisms of acne with special emphasis on Propionibacterium acnes and related therapy. Acta Derm Venereol. 2003;83(4):241-248. doi:10.1080/00015550310016463 


\section{Publish your work in this journal}

Clinical, Cosmetic and Investigational Dermatology is an international, peer-reviewed, open access, online journal that focuses on the latest clinical and experimental research in all aspects of skin disease and cosmetic interventions. This journal is indexed on CAS.

The manuscript management system is completely online and includes a very quick and fair peer-review system, which is all easy to use. Visit http://www.dovepress.com/testimonials.php to read real quotes from published authors.

Submit your manuscript here: https://www.dovepress.com/clinical-cosmetic-and-investigational-dermatology-journal 